\title{
Origin of Crashes in 3 US stock markets: Shocks and Bubbles
}

\author{
Anders Johansen \\ Teglgårdsvej 119 \\ 3050 Humlebæk, Denmark \\ email: anders-johansen@get2net.dk
}

November 10, 2018

\begin{abstract}
This paper presents an exclusive classification of the largest crashes in Dow Jones Industrial Average (DJIA), SP500 and NASDAQ in the past century. Crashes are objectively defined as the top-rank filtered drawdowns (loss from the last local maximum to the next local minimum disregarding noise fluctuations), where the size of the filter is determined by the historical volatility of the index. It is shown that all crashes can be linked to either an external shock, e.g., outbreak of war, or a log-periodic power law (LPPL) bubble with an empirically well-defined complex value of the exponent. Conversely, with one sole exception all previously identified LPPL bubbles are followed by a top-rank drawdown. As a consequence, the analysis presented suggest a one-to-one correspondence between market crashes defined as top-rank filtered drawdowns on one hand and surprising news and LPPL bubbles on the other. We attribute this correspondence to the Efficient Market Hypothesis effective on two quite different time scales depending on whether the market instability the crash represent is internally or externally generated.
\end{abstract}




\section{Addressing the Problem}

The characterization of large negative moves on the stock market is by definition of profound importance to risk management of investment portfolios. An objective non-arbitrary definition of "large" or similarly "a crash" has yet to be agreed upon by the financial community. Here, such a definition of a crash is presented in terms of $\epsilon$-drawdowns, i.e., noise filtered drawdowns where the size of the filter $\epsilon$ is determined by the historical volatility of the index. An $\epsilon$-drawdown classifies as a crash if its rank $i \sim 1$, i.e., the special name "crash" is reserved for the "few" largest negative price drops. Ideally, one would like to "prove" that these largest negative price drops are outliers to the bulk of the distribution of price drops. As for now, a large body of evidence has accumulated which suggest that it this is most likely the case $[1,2,3,4,5,6,7]$ but not more. This problem will be further addressed in a future publication and this paper only provides for an "eye-balling" analysis by the reader with figures 1-3. The reader is then left to judge for himself but should note that for the largest filter used (see figure legend), all $\epsilon$-drawdowns for all 3 indices are parametrized by the fit with the function function (4).

Specifically, for the DJIA the four largest $\epsilon$-drawdowns are characterized as crashes, for the SP500 the five largest and for the NASDAQ the six largest. Naturally, this does not exclude, e.g., that the fifth largest $\epsilon$-drawdown in the DJIA may also be characterized as a crash, but this is the interpretation of "few" put forward here. Returning to the other two indices, for the SP500 an additional $\epsilon$-drawdown has been included due to the higher historical volatility of the SP500 compared to that of the DJIA. For the same reason, the six largest $\epsilon$-drawdowns are considered for the NASDAQ. The time series extend from 01/01 1900 to 17/07 2000 for the DJIA, from 29/11 1940 to 17/07 2000 for SP500 and from 05/02 1971 to 17/07 2000 for the NASDAQ.

An $\epsilon$-drawdown is defined as a persistent decrease in the price over consecutive days from a local maximum to the next local minimum ignoring price increases in between the two of relative size less than $\epsilon$. Since they are constructed from runs of the same sign variations, $\epsilon$-drawdowns embody a rather subtle dependence which captures the way successive drops can influence each other. This "persistence" is not measured by the distribution of returns because, by its very definition, it forgets about the relative positions of the returns ${ }^{1}$. A priori, the optimal choice of filter should be bounded by $0<\epsilon \leq \sigma$, where $\sigma$ is the historical volatility, but as the noise distribution for financial data is unknown and presumably not stationary over small time scales a more qualified choice seems difficult to make.

For each of the 12 crashes considered, a causal explanation in terms of

- A surprising new piece of information as the Efficient Market Hypothesis (EMH) demands. We refer to this as a "shock".

- A prior LPPL "bubble", as defined below, to be deflated by the crash reminiscent of Adam Smith's "invisible hand".

for the crash is investigated. One may very well argue, as the author attempts to do, that the two proposed mechanisms are two sides of the same thing, however, on quite different time scales.

\section{Statistics of LPPL Bubbles}

In a series of papers, the present author with D. Sornette and co-workers $[2,4,6,8,9,10,11,12]$ have shown that on the FX, Gold and major stock markets, crashes are often preceded by precursory characteristics quantified by a log-periodic power law, specifically

$$
p(t)=A+B\left(t_{c}-t\right)^{z}+C\left(t_{c}-t\right)^{z} \cos \left(\omega \ln \left(t_{c}-t\right)-\phi\right)
$$

\footnotetext{
${ }^{1}$ Realizing this allows one to construct synthetic price data for statistical tests with the same return distribution by a reshuffling of the returns[2]
} 
The results on the US stock markets have been confirmed by several independent groups $[13,14,15]$. Eq. (1) has its origin in a Landau-expansion type of the argument and the underlying Scaling Ansatz is simply

$$
\frac{d F(x)}{d \ln x}=\alpha F(x)+\beta F^{2}(x) \ldots
$$

which to first order leads to eq. (1) with an arbitrary choice of periodic function. The concept that only relative changes are important has a solid foundation in finance, but a detailed and rigorous derivation or justification of (2) has yet to be achieved. Instead, the predictions that comes from applying this Ansatz, specifically those related to $\alpha=z+i \omega$, to the data have been compared using different markets and time periods. Specifically, assuming a Gaussian distribution it has been established empirically that

$$
\omega \approx 6.36 \pm 1.56 \quad z \approx 0.33 \pm 0.18
$$

for over thirty crashes on major financial markets [5, 6]. Hence, (1) combined with (3) is used as a definition of a LPPL bubble. Together with the concepts of "shocks", as defined above, we are lead to a consistent and coherent picture when combined with a ranking of $\epsilon$-drawdowns.

\section{Statistics of Drawdowns}

As mentioned, an increasing amount of evidence that the largest negative market moves belongs to a different population than the smaller has accumulated $[1,2,3,4,5,6,7]$. Specifically, it was found that the cumulative distributions of drawdowns on the worlds major financial markets, e.g., the U.S. stock markets, the Hong-Kong stock market, the currency exchange market (FX) and the Gold market are well parameterised by a stretched exponential

$$
N(x)=A e^{-b x^{z}}
$$

except for the $1 \%$ (or less) largest drawdowns. In general, it was found that the exponent $z \approx$ $0.7-0.9$ [3]. It is worth noting that only the distributions for the DJIA, the US\$/DM exchange rate and the Gold price exhibits "potential" outliers for the complement drawup distribution, whereas (all?) other markets shows a strong asymmetry between the tails of the drawdown and drawup distributions the latter having no outliers. This asymmetry has also been confirmed for smaller losses/gains [16]. The range of the exponent for the drawup distributions is also generally higher with $z \approx 0.9-1.1$ except for the FX and Gold markets [3].

In previous analysis and identification outliers on the major financial markets drawdowns (drawups) were either simply defined as a continuous decrease (increase) in the closing value of the price [3] or a simple filter of $1 \%$ and $2 \%$ were used $[1,5]$. Hence, in the first case a drawdown (drawup) was terminated either by any increase (decrease) in the price no matter how small. In the second case the filter was arbitrarily chosen independently of the volatility of the data. Here an $\epsilon$-drawdown is defined as follows. A local maximum in the price is identified. Then a continuation of the downward trend ignoring movements in the reverse direction smaller than $\epsilon=a \sigma$ is identified. Here, sigma is the historical volatility of the index calculated from the data and $a=1$ for the DJIA and $a=1 / 2$ for the SP500 and NASDAQ.

\section{Correspondence between Crashes, Bubbles and Shocks}

In figures 1-3 we see the cumulative $\epsilon$-drawdown distributions of the DJIA, SP500 and Nasdaq. The thresholds used were a relative threshold of $\epsilon=a \sigma$, where $\sigma$ is the historical volatility and $a$ is a number between 0 and 2, see figure legends. We see that the fits with eq. (4) for all three index fully captures the distributions except for a few cases which is referred to as "crashes". In tables 1-3 we see the ranking of the $\epsilon$-drawdowns found in the three historical indices. 
If we only note the dates of the four (DJIA), five (SP500) and six (NASDAQ) largest $\epsilon$ drawdowns in the tables, i.e., $\epsilon \neq 0,2$, we get

- DJIA: 1914, 1929, 1940 and 1987

- SP500: 1946, 1962, 1987 , 1998, 2000

- Nasdaq: $1978,1987 \star, 1998 \star, 2000$

Here $\star$ denotes two $\epsilon$-drawdown linked to the same event. Except for the crashes related to the outbreak of WWI in 1914 and WWII in 1940 quite remarkably all of these outliers have logperiodic power law precursors well-described by eq. (1) and (3). In addition, all have previously been published $[4,6]$. For the pure drawdowns, i.e., $\epsilon=0$, the ranking order is permutated and for the DJIA the 1940 event is replaced by a 1933.55 event, for the SP500 the 1978 event is replaced by a 1974.72 event and for the NASDAQ the 1978 event is replaced by a 1990.62 event. These three "new" events coincide with the political maneuvering of president F. D. Roosevelt ${ }^{2}$ upsetting the financial markets, the political turmoil caused by the resignation and the controversial pardoning of president R. Nixon on August 8th and September 8th 1974 and an $\epsilon$-drawdown of $17.8 \%$ in the Japanese Nikkei index, respectively, and should thus be classified as a shocks.

The results presented here means that the joint evidence from the ranking of $\epsilon$-drawdowns in the DJIA, the SP500 and the Nasdaq identifies all crashes with log-periodic power law precursors found in the US stock market except the crash after the LPPL bubble of 1937. Naturally, the optimal threshold (according to some specific definition) used in the outlier identification process is related to that particular index volatility. However, the volatility is again nothing but a measure of the two-point correlations present in the index, which we have proved to be an insufficient measure when dealing with extreme market events. This presumably explains this miss in the 1-to-1 correspondence between crashes on one hand and shocks and LPPL bubbles on the other.

\section{Conclusion}

The analysis presented here have strengthen the evidence for outliers in the financial markets and that the concept can be used as a objective and quantitative definition of a market crash. Furthermore, we have shown that the existence of outliers in the drawdown distribution is primarily related to the existence of log-periodic power law bubbles prior to the occurrence of these outliers or crashes. In fact, of the 12 largest drawdowns identified as outliers only 3 did not have prior log-periodic power law bubble and these 3 outliers could be linked to a specific major historical event. In complement, only 1 (1937) previously identified log-periodic power law bubble was not identified as an outlier.

the analysis presented suggest a one-to-one correspondence between market crashes defined as top-rank filtered drawdowns on one hand and surprising news and LPPL bubbles on the other. We attribute this correspondence to the Efficient Market Hypothesis effective on to quite different time scales depending on whether the market instability the crash represent is internally or externally generated.

Further work is needed to clarify the role of different coarse-graining methods as well as to arrive at a quantitative choice for $\epsilon$ based on the data. Naturally, the choice of $\epsilon$ should not only be determined by the volatility but should also depend further on the type of market.

\footnotetext{
${ }^{2}$ Roosevelt New Deal policy included the passing of the Securities Acts of 1933 and 1934 as well as going of the Gold standard in 1933 .
} 
Cited papers by the author are available from http://www.get2net.dk $/ \mathrm{kgs} /$ pub.html.

\section{References}

[1] A. Johansen and D. Sornette, Stock market crashes are outliers, European Physical Journal B 1, 141-143 (1998).

[2] A. Johansen and D. Sornette, The Nasdaq crash of April 2000: Yet another example of logperiodicity in a speculative bubble ending in a crash, Eur. Phys J. B 17 pp. 319-328 (2000).

[3] A. Johansen and D. Sornette, Large Stock Market Price Drawdowns Are Outliers, J. of Risk, vol. 4, Number 2, pp. 69-110 (2001/02)

[4] D. Sornette and A. Johansen, Significance of log-periodic precursors to financial crashes, Quantitative Finance vol.1 pp. 452-471 (2001) and references.

[5] A. Johansen, Characterization of large price variations in financial markets, Physica A 234 pp. 157-166 (2003).

[6] A. Johansen and D. Sornette, Shocks, Bubbles and Crashes in Financial Markets. Invited subm. Nova Science Publishers, issue tentatively entitled, "Contemporary Issues in International Finance", cond-mat

[7] F. Lillo and R.N. Mantegna, Symmetry alteration of ensemble return distribution in crash and rally days of financial markets, European Physical Journal B 15, pp. 603-606 (2000).

[8] D. Sornette, A. Johansen and J.P. Bouchaud, Stock Market Crashes, Precursors and Replicas, J. Phys. I. France 6 pp. 167-175 (1996).

[9] D. Sornette and A. Johansen, Large financial crashes, Physica A 245, pp. 411-422 (1997).

[10] A. Johansen and D. Sornette, Critical Crashes, RISK 12 (1), 91-94 (1999).

[11] A. Johansen, D. Sornette and O. Ledoit, Predicting Financial Crashes using discrete scale invariance, Journal of Risk 1 (4), 5-32 (1999).

[12] A. Johansen, O. Ledoit and D. Sornette, Crashes as critical points, International Journal of Theoretical and Applied Finance 3 (2), 219-255 (2000).

[13] J.A. Feigenbaum, J.A. and P.G.O. Freund, Discrete scale invariance in stock markets before crashes, Int. J. Mod. Phys. B 10, pp. 3737-3745 (1996). J.A. and P.G.O. Freund, Discrete scale invariance and the "second black Monday", Modern Physics Letters B 12, 57-60 (1998).

[14] N. Vandewalle, P. Boveroux, A. Minguet and M. Ausloos, The crash of October 1987 seen as a phase transition: amplitude and universality, Physica A 255, pp. 201-210 (1998). N. Vandewalle, M. Ausloos, Ph. Boveroux, A. Minguet, How the financial crash of October 1997 could have been predicted. European Physics Journal B 4: 139-141 (1998). N. Vandewalle, M. Ausloos, Ph. Boveroux, A. Minguet, Visualizing the log-periodic pattern before crashes, European Physical Journal B 9, 355-359 )1999).

[15] W. Paul and J. Baschnagel, Stochastic Processes : From Physics to Finance, Springer, Berlin, Heidelberg, 2000.

[16] I. Simonsen. M.H. Jensen and A. Johansen, Eur. Phys. J. Vol. 27 No. 4, p. 583 (2002). M. H. Jensen, A. Johansen and I. Simonsen, Physica A 324 pp. 338-343 (2003).

[17] A. Johansen and D. Sornette, Outlier analysis using "elastic time". In preparation. 


\begin{tabular}{|c|c|c|c|c|c|c|c|c|c|}
\hline$\epsilon$ & Date & Size & Duration & Class & $\epsilon$ & Date & Size & Duration & Class \\
\hline 0 & $1987.786 \star$ & $30.7 \%$ & 4 days & Bubble & $\sigma$ & 1914.374 & $32.7 \%$ & 64 days & Shock \\
\hline 0 & 1914.579 & $28.8 \%$ & 2 days & Shock & $\sigma$ & $1987.786 \star$ & $30.7 \%$ & 4 days & Bubble \\
\hline 0 & $1929.818 \star$ & $23.6 \%$ & 3 days & Bubble & $\sigma$ & $1929.810 \star$ & $29.5 \%$ & 6 days & Bubble \\
\hline 0 & 1933.549 & $18.6 \%$ & 4 days & Depression & $\sigma$ & 1940.261 & $23.7 \%$ & 44days & Shock \\
\hline
\end{tabular}

Table 1: List and properties of the highest ranked $\epsilon$-drawdowns for $\epsilon=0$ (left part) and $\epsilon=\sigma$ (right part) of the DJIA from 01/01 1900 to 17/07 2000. The outliers are ranked by decreasing amplitudes. The term "shock" refers to an outlier which has been triggered by an event exogenous to the market. The term "bubble" embodies the idea that the corresponding $\epsilon$-drawdown corresponds to a crash ending a speculative LPPL bubble.

\begin{tabular}{|c|c|c|c|c|c|c|c|c|c|}
\hline$\epsilon$ & Date & Size & Duration & Class & $\epsilon$ & Date & Size & Duration & Class \\
\hline 0 & $1987.784 \star$ & $28.5 \%$ & 4 days & Bubble & $\sigma / 2$ & $1987.784 \star$ & $28.5 \%$ & 4 days & Bubble \\
\hline 0 & $1962.370 \star$ & $13.7 \%$ & 9 days & Bubble & $\sigma / 2$ & $1946.636 \star$ & $16.2 \%$ & 9 days & Bubble \\
\hline 0 & $1998.649 \star$ & $12.4 \%$ & 4 days & Bubble & $\sigma / 2$ & $1962.370 \star$ & $13.7 \%$ & 9 days & Bubble \\
\hline 0 & $1987.805 \star$ & $11.8 \%$ & 3 days & Bubble & $\sigma / 2$ & $1998.649 \star$ & $12.4 \%$ & 4 days & Bubble \\
\hline 0 & 1974.721 & $11.2 \%$ & 9 days & Shock & $\sigma / 2$ & $1987.805 \star$ & $11.9 \%$ & 3 days & Bubble \\
\hline
\end{tabular}

Table 2: Same as table 1 but for the SP500 stock market index from 29/11 1940 to 17/07 2000.

\begin{tabular}{|c|c|c|c|c|c|c|c|c|c|}
\hline$\epsilon$ & Date & Size & Duration & Class & $\epsilon$ & Date & Size & Duration & Class \\
\hline 0 & $2000.268 \star$ & $25.3 \%$ & 5 days & Bubble & $\sigma / 2$ & $1987.762 \dagger$ & $27.7 \%$ & 11 days & Bubble \\
\hline 0 & $1987.784 \dagger$ & $24.6 \%$ & 5 days & Bubble & $\sigma / 2$ & $2000.268 \star$ & $25.3 \%$ & 5 days & Bubble \\
\hline 0 & $1987.805 \dagger$ & $17.0 \%$ & 5 days & Bubble & $\sigma / 2$ & $1998.630 \dagger$ & $19.2 \%$ & 9 days & Bubble \\
\hline 0 & $1998.649 \dagger$ & $16.6 \%$ & 4 days & Bubble & $\sigma / 2$ & $1998.724 \dagger$ & $18.6 \%$ & 9 days & Bubble \\
\hline 0 & $2000.374 \star$ & $14.9 \%$ & 5 days & Bubble & $\sigma / 2$ & $1987.806 \dagger$ & $17 \%$ & 5 days & Bubble \\
\hline 0 & 1990.622 & $12.5 \%$ & 6 days & Shock & $\sigma / 2$ & $1978.753 \dagger$ & $16.6 \%$ & 21 days & Bubble \\
\hline
\end{tabular}

Table 3: Same as table 1 but for the NASDAQ stock market index from 05/02 1971 to 17/07 2000. 


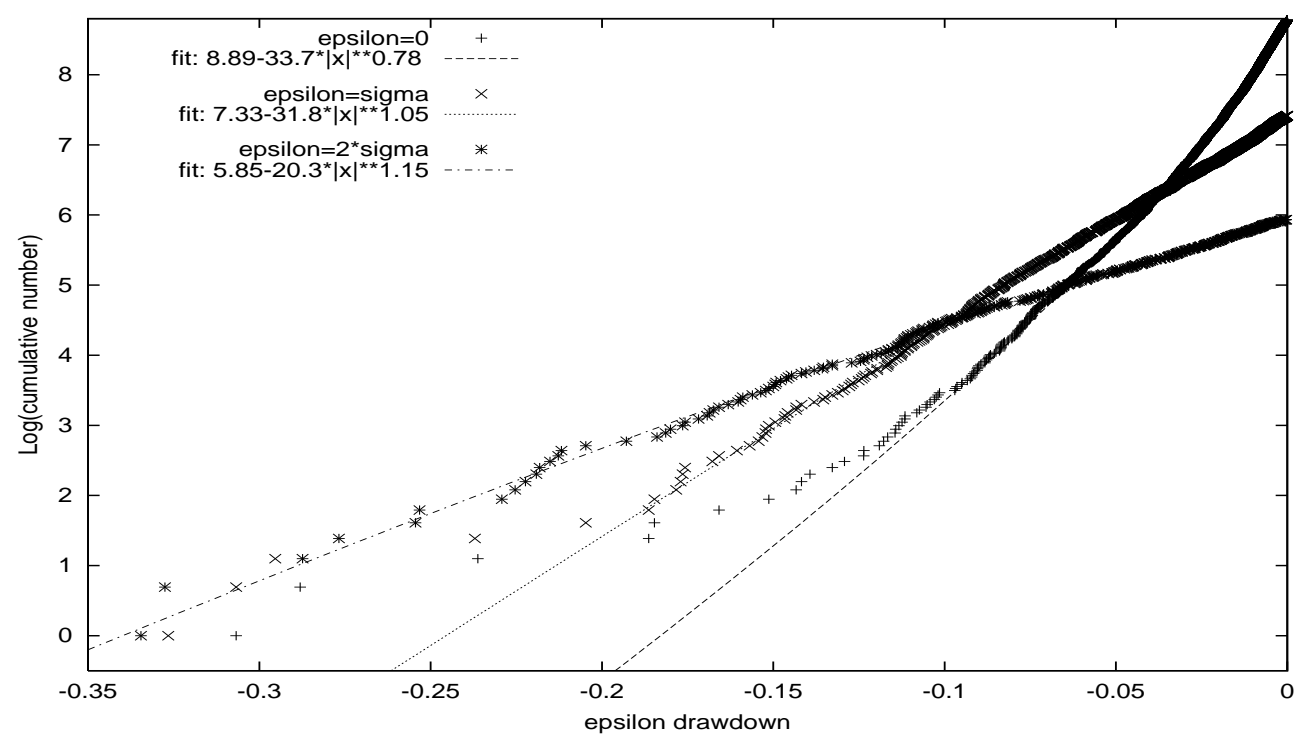

Figure 1: $\epsilon$-drawdown distribution for DJIA from 01/01 1900 to 17/07 2000.

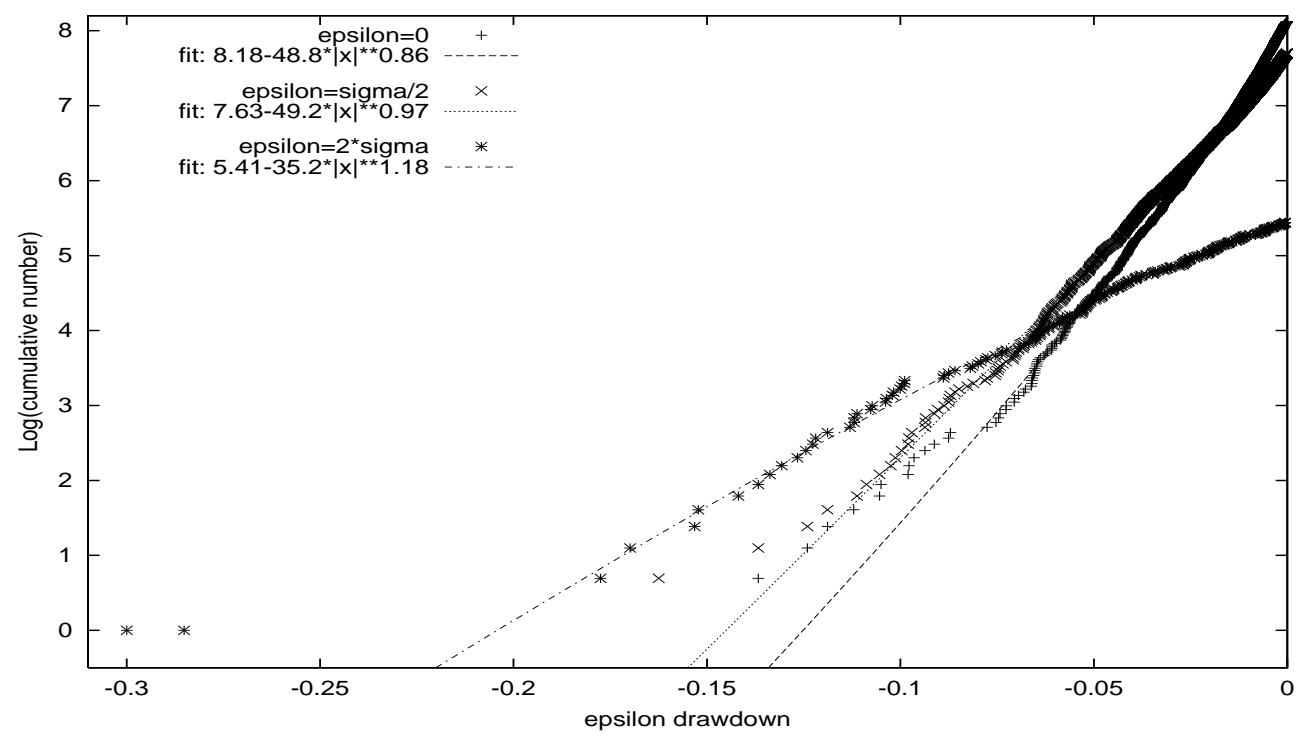

Figure 2: $\epsilon$-drawdown distribution for SP500 from 29/11 1940 to 17/07 2000.

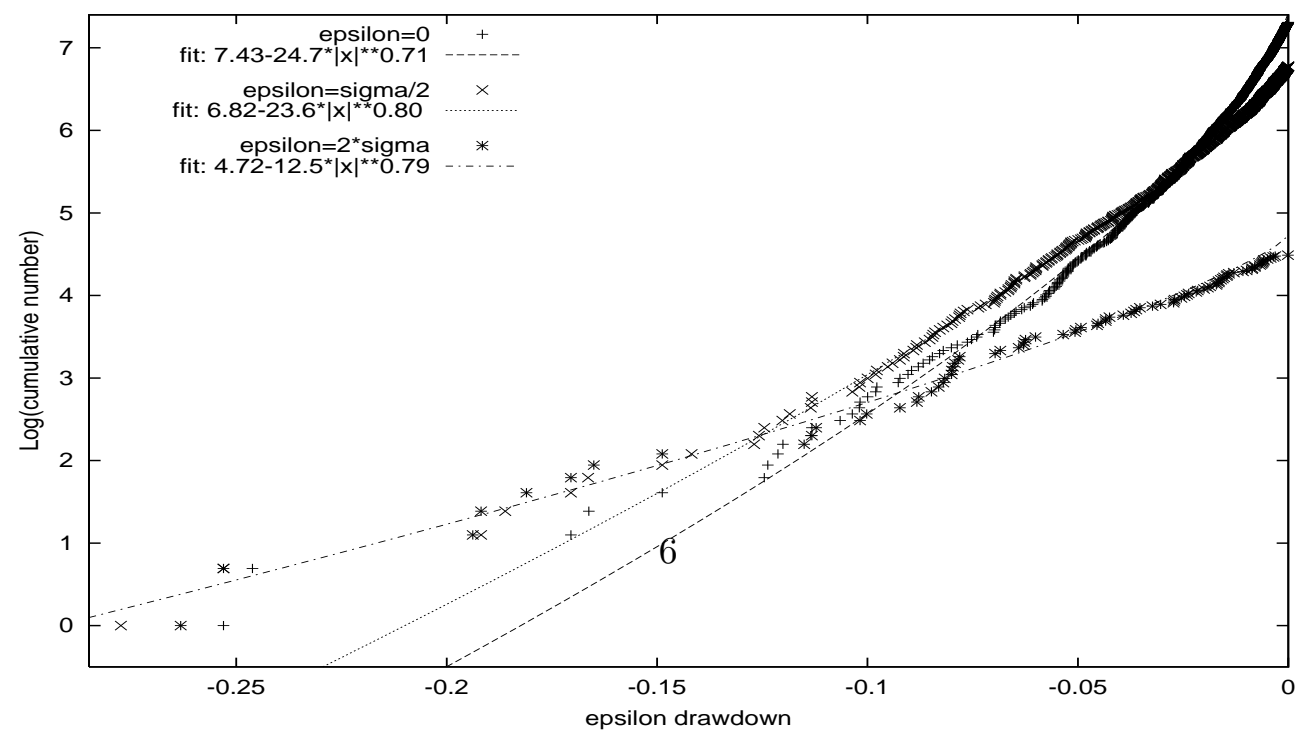

Figure 3: $\epsilon$-drawdown distribution for NASDAQ from 05/02 1971 to 17/07 2000 